\title{
Efeitos Agudos do Alongamento Estático no Desempenho da Força Dinâmica em Homens Jovens
}

\section{Acute Effects of Static Stretching in Dynamic Force Performance in Young Men}

Patrick Wander Endlich 1,2

Giovanni Rampinelli Farina2,3 Cássio Dambroz ${ }^{3}$

Washington Luiz Silva Gonçalves² Margareth Ribeiro Moysés² José Geraldo Mill ${ }^{2}$

Gláucia Rodrigues de Abreu²

1. Faculdade Salesiana de Vitória. 2. Programa de Pós-graduação em Ciências Fisiológicas, Universidade Federal do Espírito Santo, Vitória, Brasil.

3. Curso de Especialização em Bases Fisiológicas do Exercício Físico e do Desporto, Universidade Federal do Espírito Santo, Vitória, Brasil.

\section{Endereço para correspondência:} Prof. Patrick Wander Endlich Departamento de Ciências Fisiológicas, Centro de Ciências da Saúde, Universidade Federal do Espírito Santo - UFES.

Avenida Marechal Campos, 1468

- Maruípe

29040-577 - Vitória, ES - Brasil.

Tel.: +55 (27) 3335-7473

Fax: +55 (27) 3335-7330

E-mail: patrickwe@hotmail.com

Submetido em 26/08/2008 Versão final recebida em 11/12/2008 Aceito em 13/02/2009

\begin{abstract}
RESUMO
Introdução: O alongamento muscular é frequentemente utilizado nas práticas desportivas, com o objetivo de aumentar a flexibilidade muscular e amplitude articular, assim como diminuir o risco de lesões e melhorar o desempenho atlético. Objetivo: Analisar o efeito agudo do alongamento com diferentes tempos no desempenho da força dinâmica de membros superiores e inferiores em homens jovens. Métodos: Participaram da amostra 14 voluntários do sexo masculino com idade de $23 \pm 2$ anos, peso corporal de $84 \pm 10 \mathrm{~kg}$, estatura de178 $\pm 7 \mathrm{~cm}$, IMC de $26 \pm 2 \mathrm{~kg} / \mathrm{m}^{2}$ e percentual de gordura de $11 \pm 3 \%$. Eles foram avaliados com o teste de 10RM em três situações distintas: condição sem alongamento (SA), aquecimento especifico seguido do teste de 10-RM; condição com oito minutos de alongamento (AL-8), uma sessão de alongamento estático com oito minutos de duração, seguido do aquecimento e teste de 10RM; e a condição alongamento 16 minutos (AL-16), 16 minutos de alongamento seguidos dos procedimentos descritos anteriormente. Os testes foram feitos no supino reto e leg-press 45\%; os alongamentos foram selecionados de forma a atingir as musculaturas solicitadas nos respectivos exercícios. Resultados: Houve redução de 9,2\% da força muscular dinâmica de membros superiores em comparação dos grupos SA e AL16, e entre os grupos AL8 e AL16 ( $p<0,001$ ). Em membros inferiores essa redução de força ( $p<0,001)$ foi de 4,8\% para AL-8 e de 14,3\% para AL-16 em comparação com o grupo SA. Conclusão: Sessões de alongamentos estáticos efetuados antes de atividades que envolvam força dinâmica possuem a capacidade de alterar negativamente o desempenho dessa qualidade física, acarretando pior rendimento em longos períodos de alongamento.
\end{abstract}

Palavras-chave: tempo de alongamento, força muscular, exercício resistido.

\section{ABSTRACT}

Background: Muscular stretching is frequently used in sports practice with the aim to increase muscular flexibility and joint range of motion as well as to reduce injury risks and to improve athletic performance. Aim: To analyze the acute effect of stretching with different times in the dynamic strength performance of lower and upper extremities in young men. Methods: The sample was composed by 14 healthy male volunteers aged $23 \pm 2$ years, weight of $84 \pm 10 \mathrm{Kg}$, height of $178 \pm 7 \mathrm{~cm}, \mathrm{BMl}$ of $26 \pm 2 \mathrm{Kg} / \mathrm{m} 2$ and body fat of $11 \pm$ $3 \%$. They were evaluated in a 10-maximum repetition test (10-RM) in three situations: no stretching (NS); after an 8-minute session of static stretching followed by specific warm-up (SS-8); and after 16-minute and specific warm-up before 10 RM test (SS-16). Tests were performed in bench press and $45^{\circ}$ leg press exercises, and stretching was selected as to reach the musculature required in these exercises. Results: There was significant reduction $(\mathrm{p}<0.001)$ of dynamic muscular strength of upper extremities in comparison to NS with SS-16 (9.2\%) and between SS-8 (4.2\%) and SS-16 (14.3\%) to lower extremities. This difference was found in all tested conditions. Conclusion: Static stretching sessions before activities involving dynamic strength are able to negatively change performance in longer stretching periods.

Keywords: stretching time, muscular strength, physical exercise. 


\section{INTRODUÇÃO}

O alongamento muscular é frequentemente efetuado nas práticas desportivas, com o objetivo de aumentar a flexibilidade muscular e amplitude articular, assim como diminuir o risco de lesões e possivelmente melhorar o desempenho atlético (1). Todavia, quanto ao uso do alongamento imediatamente antes de sessões de exercícios resistidos (ER), nota-se controvérsia na literatura científica ${ }^{(2)}$. Shrier ${ }^{(3)}$, ao desenvolver uma revisão crítica, constatou que em 32 estudos revisados nenhum apontou que o alongamento era benéfico para 0 desempenho em eventos relacionados à força, ao torque e ao salto. Outros estudos apontam que o alongamento agudo e prolongado executado antes do exercício pode reduzir a capacidade de produzir força ${ }^{(4-9)}$ e potência ${ }^{(10-11)}$. Para explicar esses achados, especula-se que fatores neurais ${ }^{(4,5)}$ e mecânicos ${ }^{(4,12)}$ estariam envolvidos na redução temporária da atividade e da força muscular.

Por outro lado, já foi demonstrado que o treinamento de flexibilidade pode melhorar a performance nos exercícios de força envolvendo o ciclo alongamento encurtamento ${ }^{(13,14)}$, ou mesmo não exercer influência significativa no rendimento físico ${ }^{(15-17)}$. Portanto, o objetivo do presente estudo foi analisar o efeito agudo do alongamento com diferentes tempos no desempenho da força dinâmica de membros superiores e inferiores de homens jovens saudáveis.

\section{MÉTODOS}

O estudo foi conduzido segundo as normas nacionais e internacionais (Resolução 196/96-CNS e CIOMS/OMS) para pesquisas em humanos, e foi aprovado pelo Comitê Institucional de Ética (parecer no.172/2007) da Universidade Federal do Espírito Santo (UFES).

\section{Amostra}

Participaram do estudo 14 voluntários do sexo masculino, com idade de $23 \pm 2$ anos, praticantes somente de ER, no mínimo por três vezes na semana. Todos possuíam experiência prévia em ER de 12 a 18 meses. Antes da coleta de dados, os indivíduos responderam negativamente aos itens do questionário de prontidão para prática de atividade física (PAR-Q) e validaram sua participação voluntária assinando o termo de consentimento conforme resolução 196/96 para experimentos com humanos. Não foram incluídos no estudo sujeitos apresentando lesões osteomioarticulares que pudessem influenciar na realização dos testes.

\section{Desenho experimental}

A coleta de dados foi realizada em três dias não consecutivos no período de uma semana. A avaliação da força muscular foi feita com o exercício supino reto (Vitally, São Paulo-SP, Brasil) nos membros superiores e nos membros inferiores no leg-press $45^{\circ}$ (Vitally, São PauloSP, Brasil). Em um período prévio aos testes, foram aferidos o peso corporal $(\mathrm{kg})$ e a estatura $(\mathrm{cm})$ dos indivíduos em uma balança com estadiômetro (Filizola, Campo Grande-MS, Brasil), seguidos da mensuração da composição corporal (índice de massa e gordura corporal) através de um adipômetro (Sanny, São Bernardo do Campo-SP, Brasil), onde foram utilizados os protocolos propostos por Jackson e Pollock de três dobras cutâneas (peitoral, coxa e abdômen) ${ }^{(18)}$ e a equação de Siri(19).

Para avaliação da força muscular dinâmica utilizou-se o teste de 10 repetições máximas (10-RM) segundo protocolo de Baecle e Earle ${ }^{(20)}$, sob três condições experimentais distintas, sendo estas selecionadas de maneira randomizada:

Condição sem alongamento (SA): Os sujeitos realizaram aquecimento específico no supino reto e, após intervalo de dois minutos, iniciaram o teste de 10-RM. Obtida a carga no respectivo exercício, três minutos de repouso foram concedidos e a mesma sequência foi conduzida para o teste no leg-press $45^{\circ}$. O aquecimento específico consistiu em uma série de 20 repetições, a 50\% da carga de 10-RM, estimada na quilagem de treino dos indivíduos.

Condição com oito minutos de alongamento (AL-8): Os sujeitos executaram uma sessão de alongamento estático para os membros superiores com duração de oito minutos. Compondo essa sessão, três exercícios foram selecionados e enfatizaram a musculatura dos peitorais, deltoides e tríceps; em cada um eram realizadas três séries de 30 segundos, com intervalo de 30 segundos entre as séries e os exercícios. Terminado o alongamento, o indivíduo executava o aquecimento específico e, após dois minutos de intervalo, o teste de 10-RM no supino reto. Sequencialmente, foram dados três minutos de repouso e iniciada uma nova sessão de alongamento, sob as mesmas condições citadas anteriormente, porém, com exercícios para a musculatura anterior e posterior da coxa, seguidos de aquecimento específico e teste de 10RM no leg-press $45^{\circ}$.

Condição com 16 minutos de alongamento (AL-16): Os indivíduos realizaram a mesma sequência metodológica que em AL-8, porém, foram acrescidos um exercício e uma série em cada exercício nas sessões de alongamento, tanto para o membro superior quanto para o inferior, em virtude da maior duração estipulada para o alongamento. Em todas as sessões de alongamento, a sensação de desconforto muscular foi utilizada como parâmetro para regular a intensidade do exercício.

Visando reduzir a margem de erro no teste de 10-RM foram adotadas as seguintes estratégias: 1) instruções padronizadas foram oferecidas antes dos testes, de modo que o avaliado estivesse ciente de toda a rotina que envolveria a coleta de dados; 2) o avaliado foi instruído sobre a técnica de execução do exercício; 3) o avaliador esteve atento quanto à posição adotada pelo sujeito no momento da medida, pois pequenas variações no posicionamento das articulações envolvidas na ação poderiam recrutar outros músculos. No decorrer dos testes, os indivíduos foram motivados verbalmente para que chegassem à falha concêntrica em cada tentativa.

\section{Análise dos dados}

Os dados são apresentados como média \pm desvio padrão (DP). Para verificar as diferenças entre os valores das cargas encontradas nos testes de 10-RM em ambos os exercícios, foi aplicada ANOVA uma via para medidas repetidas, seguido do teste post-hoc de Tukey. O nível de significância mínimo adotado foi de $95 \%(p<0,05)$.

\section{RESULTADOS}

Na tabela 1 estão apresentadas medidas antropométricas e de composição corporal de homens jovens saudáveis (amostragem).

Tabela 1. Características antropométricas e composição corporal da amostra

\begin{tabular}{l|c|c}
\hline \multicolumn{1}{c|}{ Variáveis } & $\mathbf{N}=14$ & Min.- máx. \\
\hline Idade (anos) & $23 \pm 3$ & $18-27$ \\
\hline Peso corporal (kg) & $84 \pm 10$ & $67-110$ \\
\hline Estatura (cm) & $178 \pm 7$ & $163-188$ \\
\hline IMC (kg/cm $\left.{ }^{2}\right)$ & $26 \pm 3$ & $20-31$ \\
\hline Gordura corporal (\%) & $12 \pm 3$ & $6-18$ \\
\hline
\end{tabular}

Valores expressos em média \pm desvio padrão (DP). IMC = índice de massa corporal. 
A figura 1A mostra a redução $(p<0,01)$ progressiva no desempenho da força muscular dinâmica (carga máxima) de membros superiores quando comparada com a condição SA com AL-16 (9,2\%), que não foi observada quando comparada com a condição SA com AL-8 (0,78\%).

Em relação aos membros inferiores há redução adicional da carga máxima concêntrica ao aumentar o tempo de alongamento estático de oito (4,2\%) para 16 (14,3\%) minutos. A influência do tempo de alongamento foi mais evidente no teste de 10-RM de membros inferiores $(p<0,01)$ conforme observado na figura 1B.
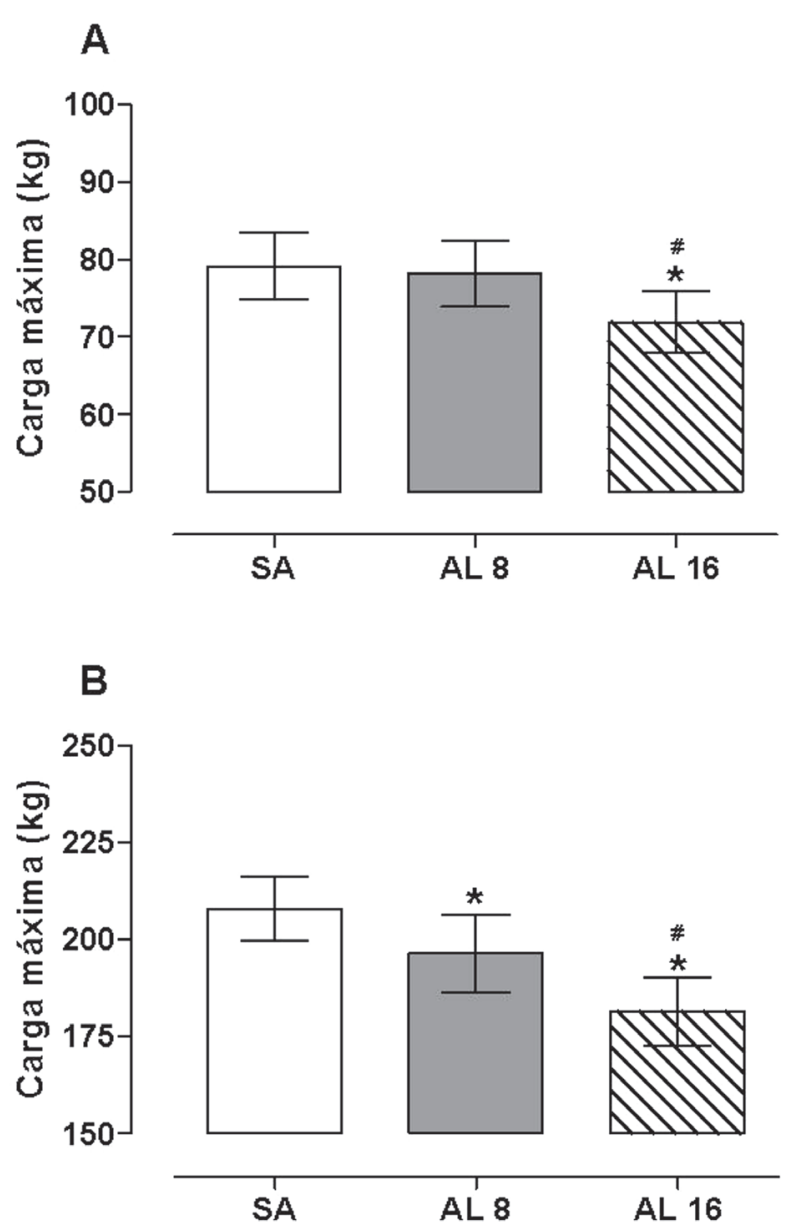

Figura 1. Determinação da carga máxima concêntrica para membros superiores (A) e inferiores (B) no teste de 10 repetições máximas (10-RM) em homens jovens saudáveis sem alongamento (SA), alongamento de 8 min. (AL-8) e 16 min. (AL-16). Valores expressos como média \pm desvio padrão (DP). ANOVA uma via, seguida do teste de Tukey como post-hoc. Diferenças significativas estabelecidas quando $p<0,01$ *vs. SA e \# vs. AL-8, $(\mathrm{N}=14)$.

\section{DISCUSSÃO}

Nosso estudo procurou analisar a influência da prática de uma sessão de alongamento estático anteriormente à execução de exercícios resistidos, onde buscamos, através da divisão do tempo das sessões de treino e da divisão dos grupos musculares, aproximar-nos da prática usual adotada na maioria dos centros de treinamento voltados para a população em geral. Tais centros trabalham, em geral e de forma simultânea, essas duas qualidades físicas. Outros trabalhos que exploraram essa temática foram realizados com tempos de alongamento mais prolongados que, normalmente, não são factíveis de ser praticados dentro da realidade de trabalho em academias ${ }^{(4,8,24)}$.
Neste trabalho mostrou-se redução significativa na carga máxima alcançada no teste de 10-RM, em membros superiores, quando precedido por sessões longas (maiores que oito minutos) de alongamento. Similarmente a esses achados, Arruda et al. ${ }^{(9)}$ encontraram diminuição no número de repetições máximas no teste 10-RM, no supino reto, em um grupo que realizava 10 minutos de alongamento estático previamente à execução do teste proposto. Na investigação da força isométrica de preensão manual, Knudson e Nofall(21) obtiveram queda significativa de 88,8\%, quando eram efetuados 100 segundos de alongamento para essa musculatura.

Com relação aos membros inferiores, ambas as condições experimentais com alongamento foram capazes de reduzir significativamente a força no teste de 10-RM. O mesmo foi encontrado por Tricoli e Paulo ${ }^{(8)}$ ao investigarem o efeito agudo do alongamento estático na força máxima de membros inferiores de homens. Nesse estudo, os autores encontraram decréscimo médio de 13,8\% na carga máxima dos sujeitos que efetuavam 20 minutos de alongamento na musculatura da coxa antes do teste de 1-RM.

Independente da musculatura testada, os dados obtidos no presente estudo indicam que a redução da força muscular dinâmica precedida por uma sessão de alongamento segue tendência de se tornar uma variável tempo-dependente. Corroborando os achados de Fowles et al.(4) ao avaliar a força de contração voluntária máxima dos flexores plantares, após uma sessão de alongamento passivo de 30 minutos nessa musculatura, encontraram redução significativa de $28 \%$, que persistiu por aproximadamente 60 minutos após o término do alongamento. Assim como Fowles et al., Marek et al. ${ }^{(22)}$ encontraram diminuição no pico de torque e na força muscular isocinética, quando homens e mulheres efetuaram protocolos de alongamento estático e de facilitação neuromuscular proprioceptiva (FNP) antes dos testes de força.

Postula-se que mecanismos neurais estariam envolvidos na redução da força muscular quando esta é precedida por alongamento estático com longa duração. Fowles et al.(4) concluíram em seu estudo que a diminuição da força muscular estaria associada à redução no recrutamento de unidades motoras, ativação dos órgãos tendinosos de Golgi e contribuição dos nociceptores. Para Achour (23), tempo prolongado de alongamento determina acomodação das fibras, de forma a comprometer a transmissão de mensagens motoras, ocasionando deformação nos componentes plásticos musculares e redução do tônus muscular. Adicionalmente aos mecanismos neurais, questões mecânicas estariam envolvidas nesses achados, onde modificações na relação comprimento-tensão com alteração da sobreposição fisiológica entre os filamentos de actina e miosina já foram demonstrados ${ }^{(4-5)}$. Na mesma linha de pensamento, estudos conduzidos por Avela et al.(24) analisaram as respostas mecânicas e neurais do gastrocnêmio e sóleo após uma hora de alongamento, e observaram redução na atividade muscular de 10,4\% e 7,6\% nesses músculos, respectivamente, e modificação no sistema tendãoaponeurose.

Por outro lado, Behm et al. ${ }^{(17)}$ investigaram o efeito agudo do alongamento estático e do aquecimento para o membro inferior, na força, equilíbrio estático, propriocepção, tempo de reação e tempo de movimento. Nesse estudo, a condição experimental determinava efetuar alongamentos estáticos intermitentes, com 135 segundos de duração para cada exercício, abrangendo a musculatura da coxa e da perna, anteriormente aos testes de força. Os autores concluíram que, embora a força tenha diminuído, tal decremento não foi suficiente para alcançar significância estatística. 
Da mesma forma, Simão et al.(14) não encontraram diferenças no resultado do teste de 1-RM no supino reto, quando precedido por sessão de FNP com seis segundos de sustentação. E, ainda, contrariando os achados do presente estudo, é possível estabelecer que, em certas atividades que envolvam o ciclo alongamento- encurtamento, complacência da unidade músculo tendínea será capaz de proporcionar energia potencial aos componentes elásticos da musculatura, beneficiando assim o desempenho ${ }^{(24)}$.

Diante dos resultados encontrados no presente trabalho, acrescidos dos achados da literatura, fica evidente que a prática prolongada do alongamento estático deve ser desencorajada quando, posteriormente, forem executadas atividades que requeiram um componente de alto rendimento para força muscular dinâmica. Assim, o alongamento não deve ser prescrito sem finalidades específicas e justificáveis nas sessões de aquecimento, como comumente pode se observar nos centros de treinamento físico; isso porque a duração do alongamento pode exercer importante efeito no desempenho físico testado tempos após.

Vale considerar que, a exemplo de outros estudos, há limitações na análise deste trabalho. Não foi realizada a quantificação do tempo de execução de cada exercício e, como os três testes foram feitos ao longo de uma semana, fadiga e dor muscular poderiam ter comprometido a eficácia na realização dos exercícios. Além disso, fatores de cunho psicológico, como motivação para execução dos testes e habituação, sempre devem ser considerados como relevantes ao se avaliar o desempenho muscular em voluntários. Entretanto, nossos resultados demonstram claramente que tempos prolongados de alongamento afetam direta e agudamente o desempenho de força. Sendo assim, essa variável obrigatoriamente deve ser considerada no planejamento das sessões de exercícios, onde as variáveis força e flexibilidade são trabalhadas simultaneamente.

\section{AGRADECIMENTOS}

Os autores agradecem ao CNPq pelo auxílio financeiro e à Academia Ritmus por ceder suas instalações para a realização do presente estudo.

Todos os autores declararam não haver qualquer potencial conflito de interesses referente a este artigo.

\section{REFERÊNCIAS BIBLIOGRÁFICAS}

1. Safran MR, Seaber AV, Garret WE. Warm-up and muscular injury prevention: an update. Clin J Sport Med 1989:8:239-49.

2. Ramos GV, Santos RR, Gonçalves A. A influência do alongamento sobre a força muscular: uma breve revisão sobre as possíveis causas. Rev Bras Cinean Des Humano 2007;9:203-6.

3. Shrier I. Does stretching improve performance? A systematic and critical review of the literature. Clin J Sport Med 2004;14:267-73.

4. Fowles JR, Sale DG, MacDougall M. Reduce strength after passive stretch of the human plantar flexores. J Appl Physol 2000;89:1179-88.

5. Avela J, Kyrolainen H, Komi PV. Altered reflex sensitivity after repeated and prolonged passive muscle stretching. J Appl Physiol 1999;86:1283-91.

6. Kokkonen J, Nelson AG, Corwell A. Acute muscle stretching inhibits maximal strength performance. Res Q Exerc Sport 1998;69:411-5.

7. Nelson AG, Corwel A. Inhinition of maximal voluntary isocinetic torque production following stretching is velocity - specific. J of Strengh and Cond Res 2001 b;15:241-6.

8. Tricoli V, Paulo AC. Efeito agudo dos exercícios de alongamento sobre o desempenho de força máxima. Rev Atividade Física Saúde 2002;7:6-13

9. Arruda FB, Faria LB, Silva W, Simão R, Senna GW, Novaes J, et al. A influência do alongamento no rendimento do treinamento de força. Rev Treinamento Desportivo 2006;7: 1-5.

10. Conwell A, Nelson AG, Sidaway B. Acute effects of passive muscle stretching on vertical jump performance. J Human Mov Stud 2001;40:307-24.

11. Yong WB, Behm DG. Effects of running, static stretching and pratice jumps on explosive force production and jumping performance. J Sport Med Phys Fitness 2003;34:119-24.

12. Kubo K, Kanehisa H, Kawakami Y, Fukunagat T. Influence of static stretching on viscoelastic properties of human tendon structures in vivo. J Appl Physiol 2001;90:520-7.

13. Wilson G, Elliot B, Wood G. Stretching shorten cycle performance enhancement through flexibility training. Med Sci Sports Exerc 1992;24:116-23.
14. Barbanti VJ, Ugrinowitsch C. O ciclo de alongamento e encurtamento e a "performance" no salto vertical. Rev Paul Educ Fis 1998;12:85-94.

15. Simão R, Giacomini MB, Dornelles TS, Marramon MG, Viveiros LE. Influência do aquecimento específico e da flexibilidade no teste de 1 RM. Rev Bras Fisiol Exerc 2003;2:134-40.

16. Young W, Elliot S. acute effects of static stretching, proprioceptive neuromuscular facilitation stretching, and maximum voluntary contractions on explosive force production and jumping performance. Res Q Exerc Sport 2001;72:273-9.

17. Behm DG, Bambuery A, Cahill F, Power K. Effect of acute static stretching on force, balance, reaction time an movement time. Med Sci Sports Exerc 2004;36:1397-402.

18. Jackson AS, Pollock ML. Generalized equations for predicting body density for men. Br J Nutr 1978:40:497-504.

19. Siri WE. Body composition from fluid spaces and density. Washington: National Academy of Science, 1961.

20. Baecle TR, Earle RW. Essential of strength training and conditioning. Champaing: Human Kinetics, 2000.

21. Knudson D, Noffal G. Time course of stretch-induced isometric strength deficits. Eur J Appl Physiol 2005;94:348-51.

22. Marek SM, Cramer JT, Fincher L, Massey LL, Dangelmaier L, Purkayastha S, et al. Acute effects of static and proprioceptive neuromuscular facilitation stretching on muscle atrength and power output. J Athl Train 2005;40:94-103.

23. Achour JÁ. Alongamento e aquecimento: aplicabilidade na performance atlética. Rev Assoc Prof Educ Fís Londrina 1995;10:50-69.

24. Avela J, Finini T, Liikavainio T, Niemelã E, Komi V. Neural and mechanical responses of the triceps surae muscle groupe after $1 \mathrm{~h}$ of reated fast passive stretches. J Appl Physiol 2004;96:2325-32.

25. Witvrouw E, Mahieu N, Danneels L, Mcnair P. Stretching and injury prevention: na obscure relationship. Sports Med 2004;34:443-9. 